\title{
Doença de Crohn metastática sem manifestação clínica intestinal ${ }^{*}$
}

\author{
Metastatic Crohn's disease without intestinal clinical manifestation
}

\author{
Marcelo D'Ambrosio Fernandes ${ }^{1}$ \\ Rosângela Deliza ${ }^{3}$ \\ Angela Cristina Bortoncello5
}

\author{
Helena D'Ambrosio Fernandes ${ }^{2}$ \\ Caio Eduardo Ferreira Pires ${ }^{4}$
}

\begin{abstract}
Resumo: Os autores relatam caso de paciente feminina de 47 anos, com lesões cutâneas ulceradas, disseminadas, iniciadas sete anos antes do acompanhamento. Os achados histopatológicos de tais lesões, somados aos endoscópicos, permitiram o diagnóstico de uma entidade rara: a doença de Crohn metastática. O valor deste diagnóstico é ainda maior, por não se encontrar na literatura, relato de caso com atividade da doença de Crohn restrita às lesões cutâneas, sem a observação de queixas digestivas expressivas.

Palavras-chave: Doença de Crohn; Fístula; Granuloma

Abstract: The authors report the case of a 47-year-old female patient with dispersed ulcerated skin lesions that appeared 7 years before medical follow-up. Histopathological and endoscopic findings of such lesions led to the diagnosis of a rare disorder: metastatic Crohn's disease. This diagnosis is even more relevant because there is no case report in the literature of Crohn's disease restricted to cutaneous lesions, without major gastro-intestinal complaints.

Keywords: Crohn disease; Fistula; Granuloma
\end{abstract}

\section{INTRODUÇÃO}

As úlceras cutâneas traduzem perda tecidual circunscrita, geralmente, de epiderme e derme, podendo haver comprometimento eventual da hipoderme. Podem ter etiologia traumática, vascular, infecciosa ou inflamatória. Na propedêutica clínica diagnóstica de lesões ulcerosas dermatológicas, é imperativo que se proceda a avaliação sistêmica do paciente, pois, muitas vezes, estamos diante da manifestação cutânea de patologia com acometimento primário não-cutâneo.

Assim acontece em úlceras relacionadas às vasculites, às micoses profundas, às micobacterioses e à doença de Crohn, entidade de lesão primária de trato digestivo, mas que pode determinar manifestações cutâneas, por contiguidade do acometimento intestinal ou não. ${ }^{1}$

Por vezes, este acometimento sistêmico não se evidencia em sinais e sintomas, é discreto ou está mascarado, mas não deve ser desvalorizado. Na doença de Crohn, é clássico o distúrbio intestinal que cursa, em casos graves, com dor abdominal, diarreia muco-sanguinolenta, emagrecimento e, por vezes, crises de abdômen agudo secundário às aderências intra-abdominais. ${ }^{2}$ Porém, muitas vezes, o acometimento não atinge tal magnitude clínica e as queixas digestivas podem ser bem menos expressivas. A colonoscopia,

\footnotetext{
Recebido em 17.9.2008.

Aprovado pelo Conselho Consultivo e aceito para publicação em 9.07.09

* Trabalho realizado em clínica particular de dermatologia - Campinas (SP), Brasil.

Conflito de interesse: Nenhum / Conflict of interest: None

Suporte financeiro: Nenhum / Financial funding: None

Médico Especialista em Dermatologia, ex-residente do Complexo Hospitalar Padre Bento de Guarulhos - Guarulhos (SP), Brasil.

Médica Especialista em Dermatologia, ex-residente do Hospital das Clínicas da Universidade Estadual de Campinas (UNICAMP) - Campinas (SP), Brasil.

Médica Patologista, com especialização em patologia digestiva no Japão. Especialista pela Sociedade Brasileira de Patologia (SBP). Laboratório de anatomia patológica - Campinas (SP), Brasil.

Médicos residentes de Clínica Médica do Hospital Municipal Dr. Mário Gatti - Campinas (SP), Brasil.

Médicos residentes de Clínica Médica do Hospital Municipal Dr. Mário Gatti - Campinas (SP), Brasil.

(C)2009 by Anais Brasileiros de Dermatologia
} 
em qualquer uma das possibilidades, aparece como método diagnóstico auxiliar de grande valor, pois, mesmo nos casos de acometimento intestinal discreto, com manifestações clínicas quase ausentes, pode confirmar o diagnóstico de Doença de Crohn por critérios macroscópicos e microscópicos.

\section{RELATO DO CASO}

Mulher de 47 anos procurou atendimento médico, por apresentar, há sete anos, nódulos e placas infiltradas e ulceradas, indolores, recobertas por crostas em mama e mamilo direito, glúteo, região suprapúbica, poplítea e tornozelo esquerdo (Figuras 1, 2 e 3). Algumas lesões drenavam secreção fluída amarelada, sem eliminação de grãos. Duas delas, tiveram início após estímulo traumático - injeção intramuscular nas lesões em glúteo, e lesão suprapúbica sobre cicatriz cesárea.

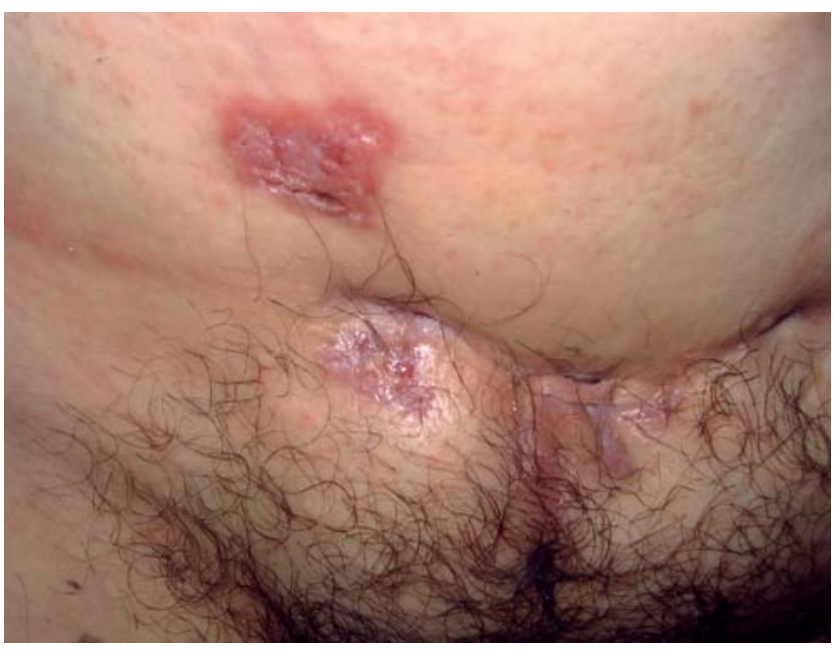

Figura 1: Lesão fistulizada em região suprapúbica

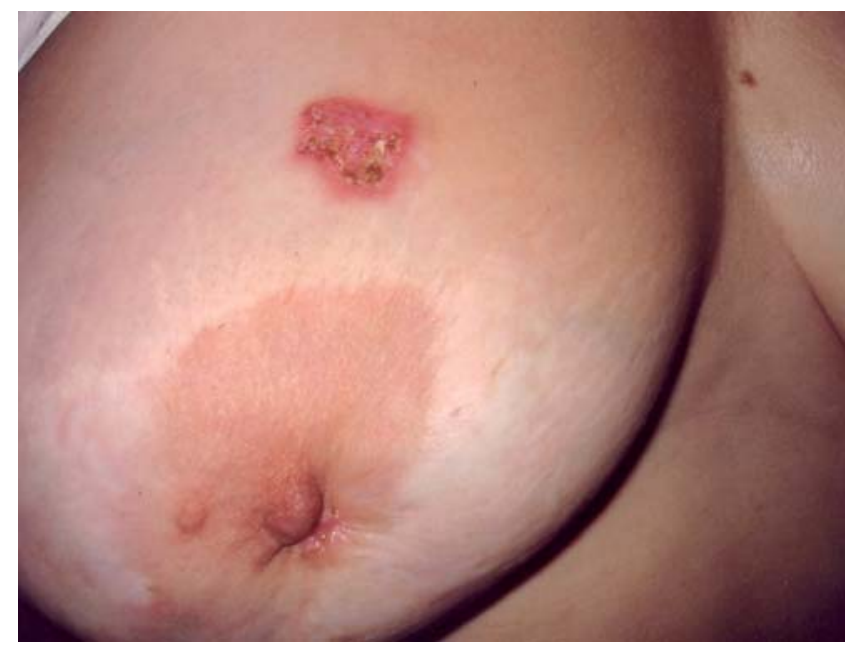

Figura 2: Lesão de mama D e retração de mamilo

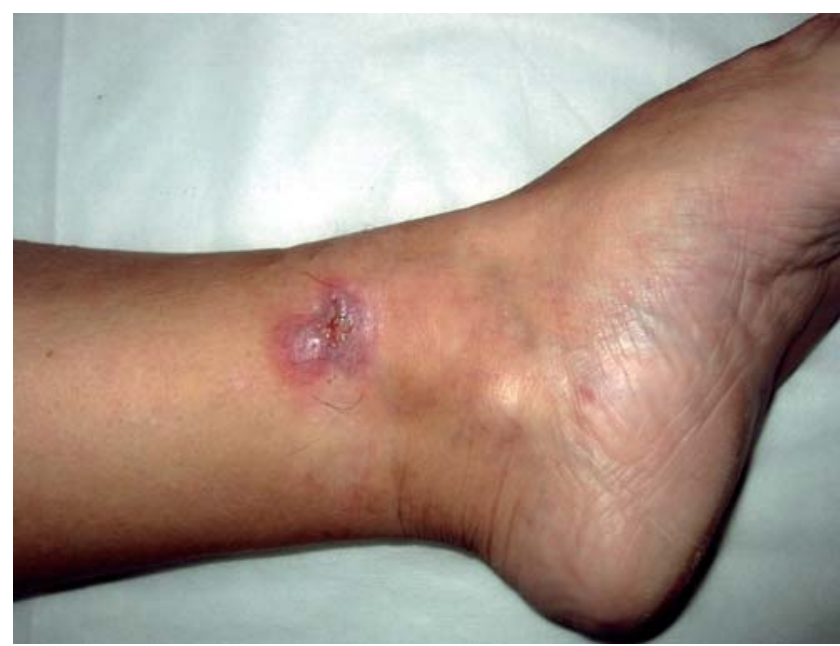

FigurA 3: Lesão nodular ulcerada em tornozelo E

Negava uso de medicações ou antecedentes mórbidos. Não apresentava febre, emagrecimento ou ulcerações orais / genitais. Sem alterações digestivas, urinárias ou antecedentes familiares relacionados. Ao exame físico, destacavam-se apenas as alterações cutâneas. A biópsia da lesão do tornozelo evidenciou processo inflamatório crônico ulcerado, com marcada reação linfo-histiocitária, sem sinais de malignidade e pesquisa para BAAR e fungos negativa. A cultura de tecido também foi negativa, para micobactérias e fungos.

Hemograma, função renal, perfil hepático, raios-X de tórax, urina 1 e complementos séricos eram normais. Sorologias para HIV, hepatite B e C, FAN e ANCA não eram reagentes. O VHS era de $29 \mathrm{~mm}$ $\left(1^{\mathrm{a}}\right.$ hora) e o PPD, não reator. A sorologia para Leishmaniose foi negativa. Solicitado colonoscopia que visualizou erosões em íleo terminal, recobertas por fibrina, com exame anatomopatológico de ileíte crônica moderada, superficialmente erosiva, sugerindo doença de Crohn (Figuras 4 e 5).

Nova biópsia de pele, agora da mama, foi então realizada com achado de processo inflamatório ulcerado e granulomatoso, sem a presença de fungos ou micobactérias e sem halo linfocitário, compatível com doença de Crohn (Figura 6). Realizou-se tratamento com mezalasina e prednisona, sem resposta. No momento, a paciente está aguardando uso de infliximabe.

\section{DISCUSSÃO}

A doença de Crohn é uma moléstia inflamatória intestinal idiopática que pode comprometer todo trato digestivo, desde a cavidade oral até a borda anal. Manifesta-se em qualquer idade, com áreas afetadas da mucosa intercalando-se com áreas sadias. Há, contudo, evidente predileção pelo íleo terminal. ${ }^{1}$

As alterações inflamatórias - percebidas pela colonoscopia na forma de eritema e edema de muco- 


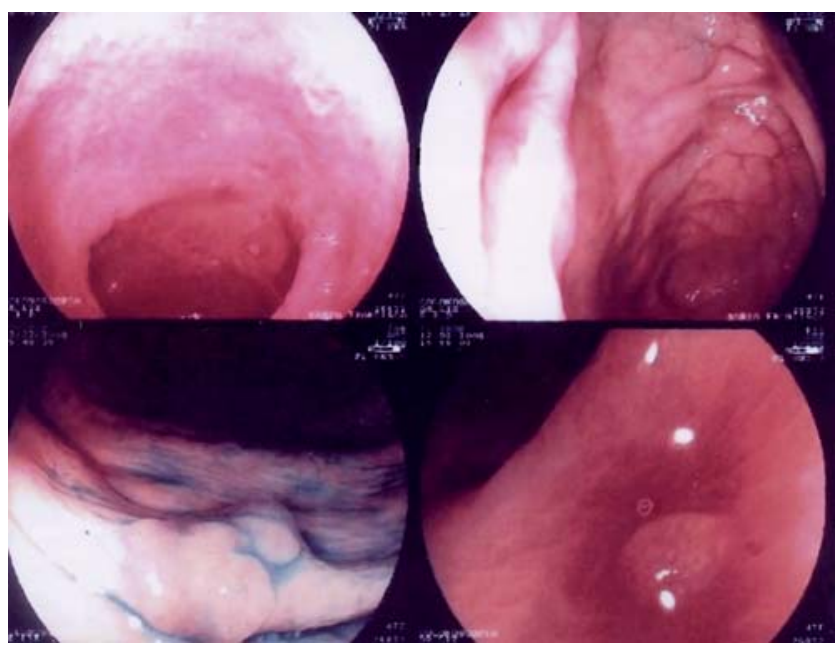

FigurA 4: Aspecto macroscópico da colonoscopia com enantema e erosões recobertas por fibrina

sa, ulcerações frequentes e formação de pseudopólipos - podem levar a estenoses e perda da mobilidade intestinal, com quadros disabsortivos e deficiências nutricionais secundárias, dentre as quais, destacamse: a deficiência de zinco (com manifestações clínicas similares à acrodermatite enteropática), a pelagra e a anemia perniciosa. ${ }^{2}$ Também está classicamente associado à sua atividade o eritema nodoso, lesões de pioderma gangrenoso e vasculites necrotizantes cutâneas. ${ }^{3}$ Há relato de doença de Crohn intestinal, associada à psoríase pustulosa aguda. ${ }^{4}$

Não raro, a doença de Crohn pode determinar lesões cutâneas específicas, na maioria das vezes, contíguas ao acometimento intestinal, apresentando-se clinicamente como fístulas ou ulcerações perianais. ${ }^{5}$ $\mathrm{Na}$ literatura descreve-se uma entidade caracterizada pelo surgimento de lesões cutâneas, com aspecto granulomatoso ao exame histopatológico, sem relação

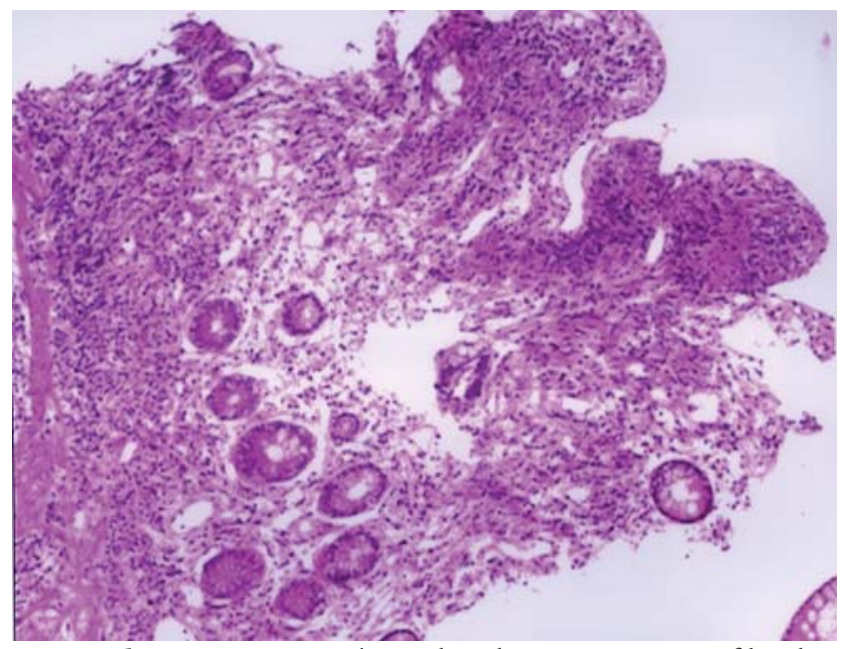

Figura 5: Aspecto microscópico da colonoscopia, com infiltrado inflamatório misto, predominantemente linfoplasmocitário, de padrão folicular, com esboço de isolado granuloma epitelióide na lâmina própria (HE, 40x)

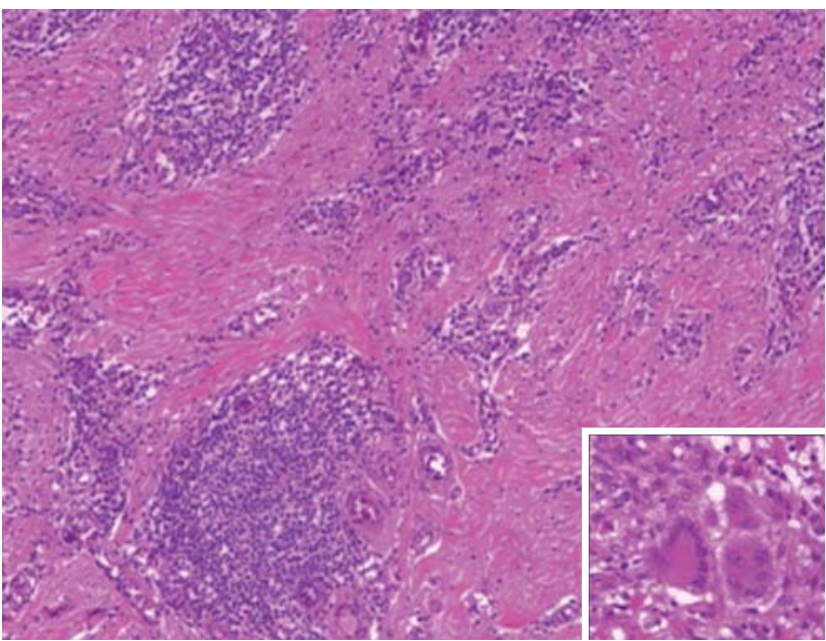

Figura 6: Aspecto microscópico de biópsia de mama, com moderado infiltrado linfoplasmohistiocitário. com formação de agregados linfoides e focos de reação granulomatosa (HE, 100x). No detalhe (HE, 400x), células gigantes multinucleadas do tipo corpo estranho e Langerhans

topográfica com o Crohn intestinal, isto é, lesões específicas separadas do acometimento intestinal, por grandes áreas de pele normal. Trata-se da chamada DOENÇA DE CROHN METASTÁTICA (DCM). ${ }^{6}$ Neste caso, há predileção pelo acometimento de genitais - o que se observa em $2 / 3$ dos casos de DCM em crianças e jovens menores de 18 anos - e também de extremidades inferiores (38\% dos casos), tronco e abdome (24\%), extremidades superiores (15\%), face e lábios (11\%) e flexuras (8\%). O acometimento generalizado no Crohn metastático não chega a ocorrer em $4 \%$ dos casos. $^{7}$ No nosso caso, a paciente apresentava comprometimento de extremidades inferiores (tornozelo e joelho), abdome inferior (região suprapúbica), tronco (mama) e quadril (glúteo).

No diagnóstico diferencial da DCM, destacamse: o pioderma gangrenoso, a micobacteriose atípica, a tuberculose cutânea, as micoses profundas, a actinomicose, a doença de Behçet, a granulomatose de Wegener e a poliarterite nodosa cutânea. ${ }^{5}$ No caso descrito, o estudo anatomopatológico, microbiológico e sorológico afastou tais hipóteses, e a solicitação da colonoscopia, apesar da ausência de dor abdominal, emagrecimento ou diarreia, buscava afastar DCM. "Com a presença de alterações macroscópicas e microscópicas da doença de Crohn intestinal, o diagnóstico seria presuntivo, não fosse à nova biópsia de pele (mama) que acusou reação granulomatosa.

Kafity publicou artigo que relaciona, em 100\% das vezes, a presença de doença intestinal à DCM. ${ }^{7}$ Em nosso caso, a doente apresentava atividade inflamatória moderada no íleo, sem manifestação clínica. Entretanto, a atividade da doença estava evidente nas 
lesões cutâneas.

A doença de Crohn cutânea tende a ter curso crônico, com o tratamento da doença gastrointestinal, clareando algumas lesões de pele. A remoção cirúrgica da porção afetada do trato digestivo não se correlaciona diretamente com a melhora das lesões cutâneas. ${ }^{8}$

A terapia é vasta. Metronidazol, corticoide oral, sulfassalazina, tetraciclina, azatioprina e ciclosporina estão entre as opções, com resultados favoráveis relatados, mas sem respostas e tempo de tratamento padronizados. ${ }^{5}$ Os efeitos colaterais destas drogas para uso crônico limitam a prescrição. A ciclosporina parece ser útil para induzir remissões e tratar exacerbações agudas, quando os corticoides falham, mas hipertensão e insuficiência renal são comuns, a longo prazo. ${ }^{8}$

Quando não se alcança resposta clínica com as drogas citadas, a opção de uso dos biológicos - uma nova classe terapêutica com atividade anticitocinas tem se mostrado efetiva. ${ }^{9}$ Para controle da atividade inflamatória das lesões da doença de Crohn clássica, encontram-se aprovados o adalimumabe e o infliximabe. Este último apresenta ação anti TNF alfa e foi utilizado em seguimento, publicado por Cohen, em 2001, com boa tolerabilidade e controle das lesões cutâneas fistulizadas. ${ }^{9,10}$ A ação destes anticorpos monoclonais promoveu melhora clínica rápida e duradoura, nas séries publicadas. ${ }^{10}$

A publicação de Kafty, em 1993, relaciona, em $100 \%$ dos casos, a doença intestinal à DCM. Não descreve, contudo, casos de acometimento intestinal histológico puro, sem queixas clínicas evidentes, como ocorreu no caso descrito. Assim, a apresentação de um caso com atividade inflamatória clínica da doença de Crohn, limitada à pele, em paciente sem queixas intestinais expressivas, merece destaque pela necessidade de se pensar nesta entidade, como parte do diagnóstico diferencial de lesões ulceradas, mesmo que o acometimento digestivo não esteja presente.

\title{
REFERÊNCIAS
}

1. Felley C, Mottet C, Juillerat P, Froehlich F, Burnand B, Vader JP, et al. Fistulizing Crohn's disease. Digestion. 2005;71:26-8.

2. Thayu M, Shults J, Burnham JM, Zemel BS, Baldassano $\mathrm{RN}$, Leonard MB. Gender differences in body composition deficits at diagnosis in children and adolescents with Crohn's disease. Inflamm Bowel Dis. 2007;13:1121-8.

3. Burgdorf W. Cutaneous manifestations of Crohn's disease. J Am Acad Dermatol. 1981;5:689-95.

4. Fernandes EI, Ferreira TC, Silveira TR, Ferreira CT. Psoríase pustulosa associada à doença de Crohn: relato de caso. An Bras Dermatol. 2000;75:57-64.

5. Gilson RT, Elston D, Pruitt A. Metastatic Crohn's Disease: Remission induced by mesalamine and prednisone. J Am Acad Dermatol. 1999;41:476-9.

6. Shum D, Guenther L. Metastatic Crohn's disease: case report and review of the literature. Arch Dermatol. 1990; 126:645-8

7. Kafity A, Pellegrini A, Fromkes J. Metastatic Crohn's disease: a rare cutaneous manifestation. J Clin Gastroenterol. 1993;17:300-3.

8. Saschar D. Maintenance therapy in ulcerative colitis and Crohn's disease. J Clin Gastroenterol. 1995;20:117-22.

9. Farrel RJ, Shah SA, Lodhavia PJ, Alsahli M, Falchuk KR, Michetti P, et al. Clinical experience with infliximab therapy in 100 patients with Crohn's disease. Am J Gastroenterol. 2000;95:3490-7.

10. Cohen MD. Efficacy and safety of repeated infliximab infusions for Crohn's disease: 1-year clinical experience. Inflamm Bowel Dis. 2001; (Suppl 1):S17-22.

\author{
ENDEREÇO PARA CORRESPONDÊNCIA / MAILING ADDRESS: \\ Marcelo D'Ambrosio Fernandes \\ Rua Sacramento, 501 Centro. \\ 13010210 Campinas SP \\ Tel:/Fax: 19 32363224; 19 32581127; 1981117893 \\ E-mail: dermato.dambrosio@gmail.com
}

Como citar este artigo / How to cite this article: Fernandes MD, Fernandes HD, Deliza R, Pires CEF, Bortoncello AC. Doença de Crohn metastática sem manifestação clínica intestinal. An Bras Dermatol. 2009;84(6):651-4. 